\title{
Design and Test of a High-Resolution Acquisition System for Marine Seismology
}

\section{Shahram Shariat-Panahi, Francisco Corrêa Alegria, and Antoni Mànuel Làzaro}

A

ctive and passive seismology require high-resolution, low-level signal acquisition. In both methods,

the equipment most widely used to acquire seismic data in the marine environment is an ocean bottom seismometer (OBS). We designed and built a high-resolution acquisition system prototype for an OBS that is mainly used in active seismic experiments in which maps are generated of the width and material of ocean bottom sublayers. Other OBS system applications, in which the objective is to estimate the location and magnitude of an earthquake require higher autonomy. When an OBS is used in a permanent seafloor observatory with no power supply limitation, it can generate tsunami alert signals to shore.

This paper presents the design and implementation of a high-resolution, low-noise acquisition system of acoustic signals for marine seismology. We discuss the following:

Dinimizing system noise in the design and implementation stage, and

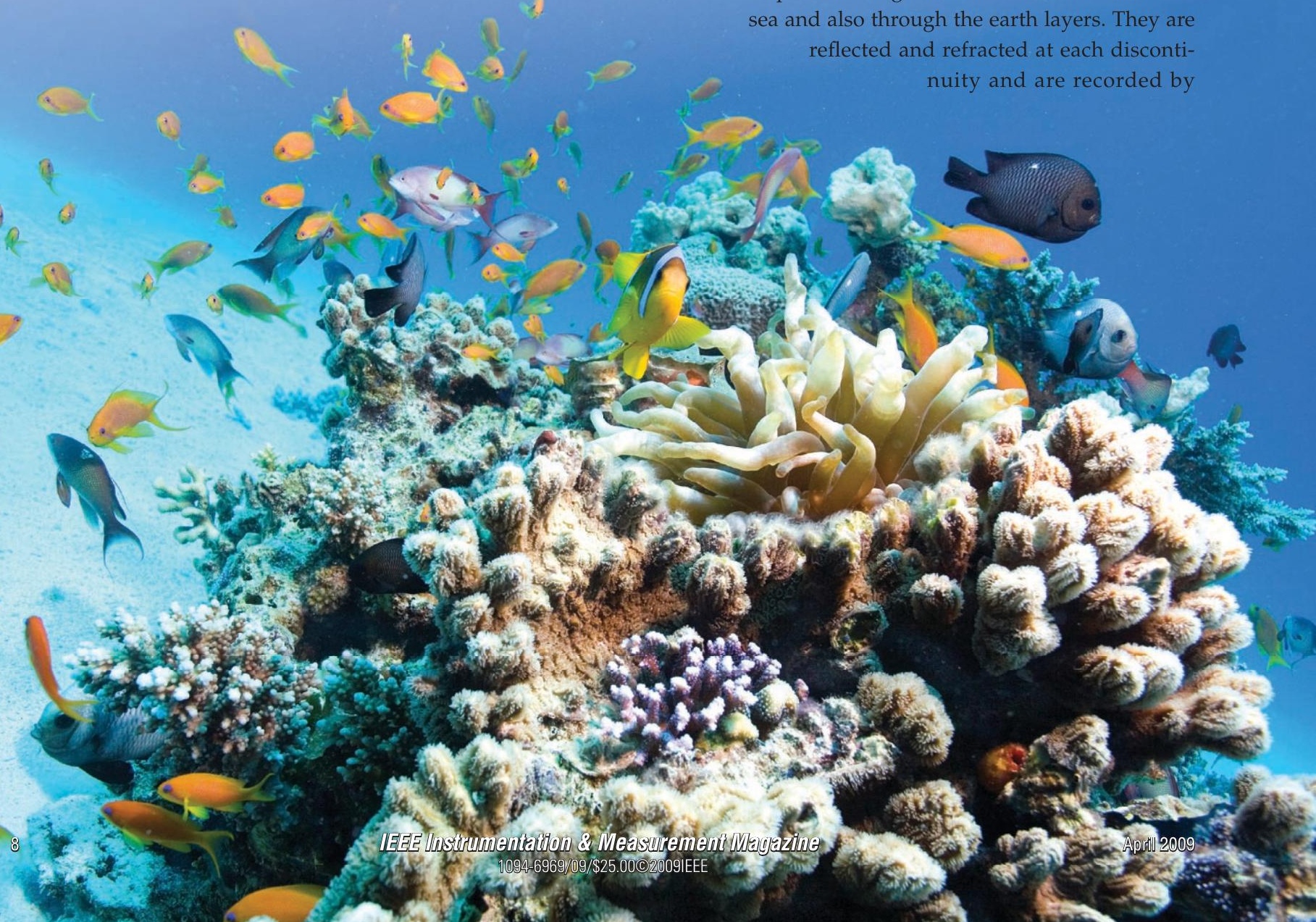

\section{Background}

In passive seismology, the nature and location of an earthquake source is unknown. Seismometers are used to record an earthquake and micro-seismic activities in the marine environment, and the acquired data are used by scientists to estimate the magnitude and location of an earthquake. In active seismology, a compressed-air gun is used as an artificial source of seismic activity and is dragged by a research vessel

during an experiment. The acoustic signals generated by the compressed-air gun travel to the bottom of the sea and also through the earth layers. They are nuity and are recorded by

finding the specifications of the system before it is used in
real environmental conditions by using a series of characterization tests based on IEEE Standard 1057 [1] and IEEE external noise coupling to the system is minimized to calculate the $S / N$ ratio, dynamic range, effective number of bits (ENOB), and analog-to-digital converter (ADC) clock jitter. These parameters help to compare data loggers designed to be used in OBSs.

reflected and refracted at each disconti- 
the acquisition system [3]. Figure 1 shows a picture of our OBS prototype.

The signal travels tens of kilometers through the earth layers, and it is a very low signal when collected by the acquisition system. The data logger $S / N$ needs to be optimized. The OBS is equipped with three sensors:

D a tri-axial geophone [4] based on three accelerometers placed at right angles (one for each axis), which couples to the seabed to collect seafloor vibrations,

- a hydrophone that senses water pressure, and

D an acoustic transducer for the recovery process.

Once the experiment is over, an acoustic signal is sent to the OBS which releases the anchor weight used for deployment, and that allows the OBS to rise to the surface.

\section{The Acquisition System Design}

The acquisition system consists of four main blocks: a microcontroller and data storage module, an ADC module, a power regulation module, and a time base module; a lithium-ion battery pack is the main power supply. Figure 2 shows a diagram of the acquisition system.

\section{Micro-Controller and Data Storage Module}

The micro-controller and storage module is based on a MC68332 microcontroller and utilizes a 4 GB CompactFlash memory card for data storage. This module is in charge of configuring the ADC module by selecting the sampling rate and low-power operations. The acquisition software acquires data continuously from input channels through a Queued Serial Peripheral Interface bus, performs time stamping by using the integrated real-time clock and stores the data in the CompactFlash memory card. Furthermore, this module performs default configuration of the system, time synchronization with a GPS signal (or by using a crystaldescribed in the next paragraph) before OBS deployment, clock drift calculation after OBS recovery, and low-power sleep.

\section{The Time Base Module}

In active marine seismology, the quality of the ocean bottom model obtained after signal processing is related directly to the

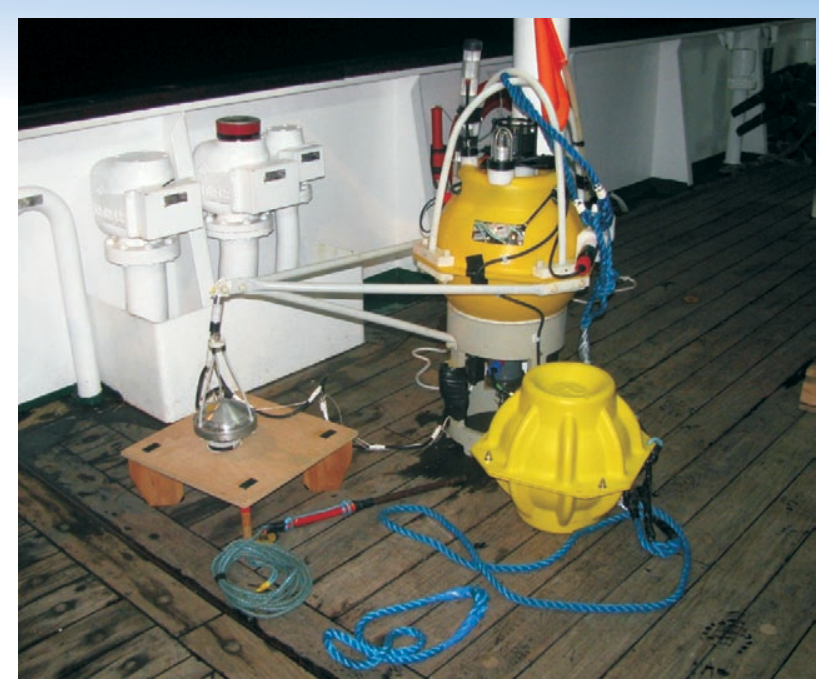

Fig. 1. Ocean bottom seismometer (OBS)

timing of the acquired data. As the equipment has no access to a GPS signal for time synchronization when it is on the seabed, a single precise crystal is used to generate all the signals necessary for data acquisition. The time base module uses a 32.768 $\mathrm{MHz}$ Vectron Temperature Compensated Crystal Oscillator as the main time reference.

\section{The Power Regulation Module}

To reduce the noise coupling from the power regulation module to the ADC module and to maximize the autonomy of the instrument, we used MAX1653 switched converters with output noise control. This regulator reduces interference due to switching noise by ensuring a constant switching frequency regardless of the load and line conditions, thus concentrating the emissions at a known frequency outside the system bandwidth.

\section{The ADC Module}

The noise level of the ADC module defines the final resolution of the overall system. Special care must be taken in designing this module in order to obtain high-quality data. The ADC

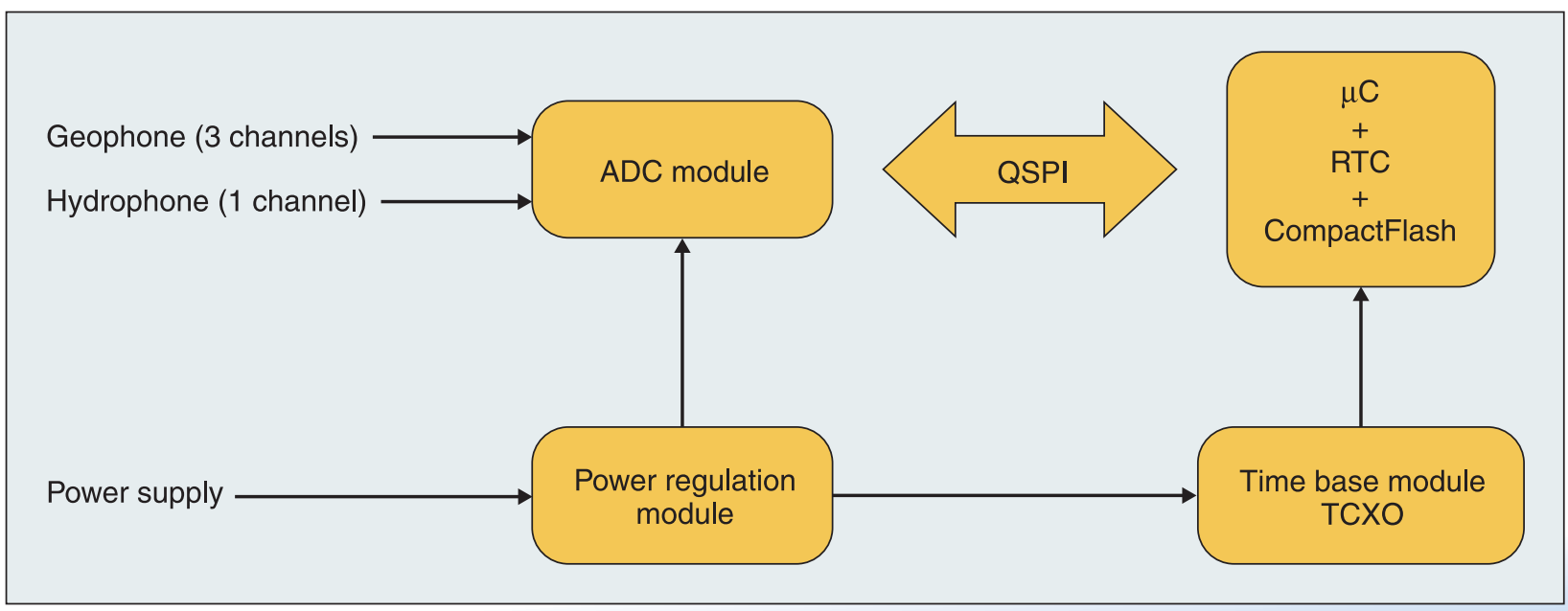

Fig. 2. Acquisition system block diagram. 


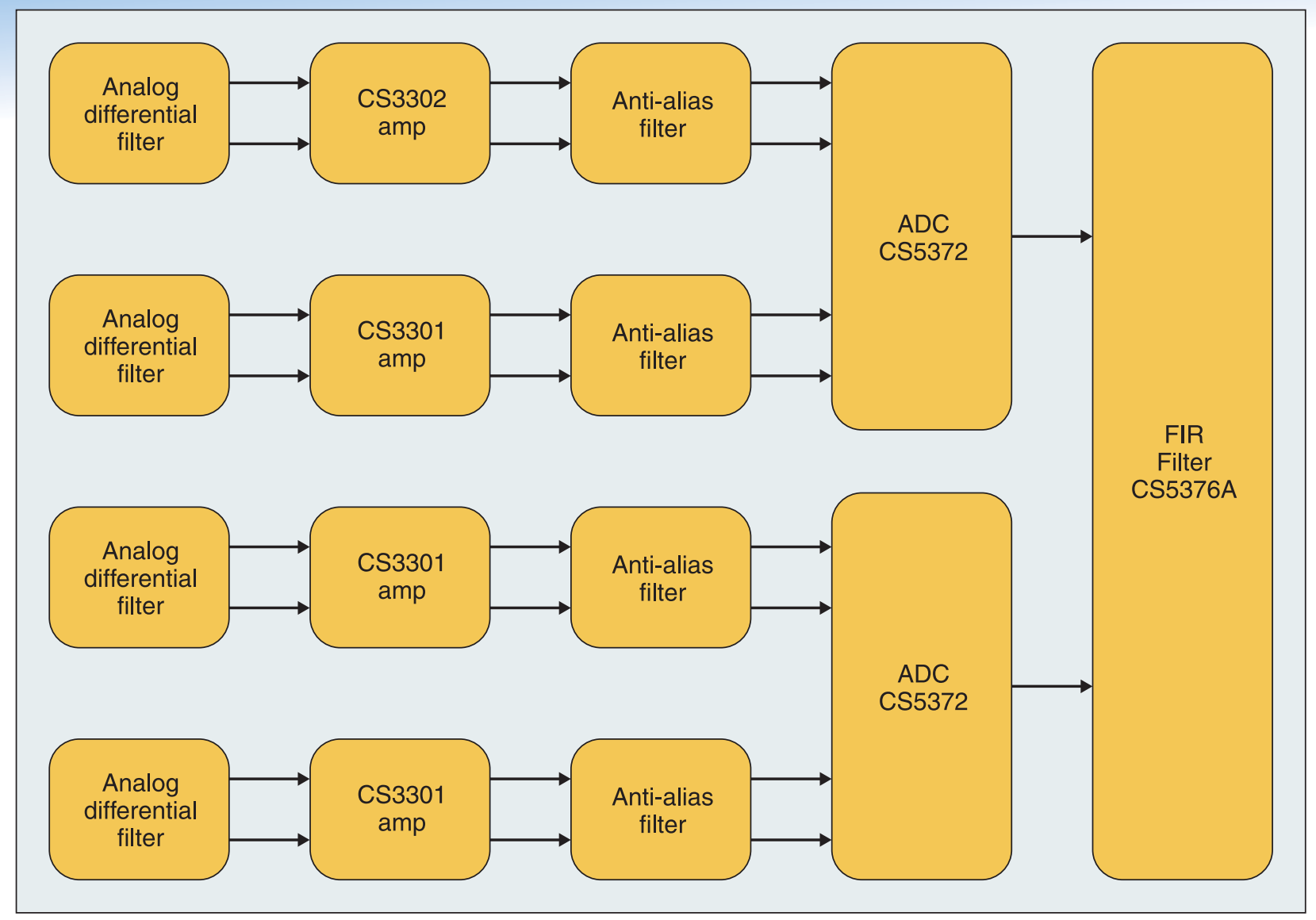

Fig. 3. Block diagram of the ADC module.

module is based on a four-channel, 24-bit CS5372 ADC oversampling at $512 \mathrm{kHz}$ and a CS5376A digital filter for data decimation and interfacing with the microcontroller module. The CS5372s are two-channel, high-dynamic range, fourth-order $\Delta-\Sigma$ modulators that provide a dynamic range of $130 \mathrm{~dB}$ at $103-$ $\mathrm{Hz}$ bandwidth and a total harmonic distortion (THD) of -118 dB. Figure 3 shows the block diagram of the ADC module.

The acquisition systems used for active seismic surveys require a low noise level, thus maximizing the ENOB as well as the dynamic range. Electromagnetic interference (EMI) and channel crosstalk are common causes of signal degradation in high-resolution acquisition systems. Poor design of the ADC module results in a high noise level and therefore a low $S / N$. The following considerations were taken into account in the design of the ADC module in order to obtain the required noise performance.

Analog stage optimizations: The input stage of the ADC module is based on the CS3301/02 low-noise, fully differential amplifiers with a noise level of $380 \mathrm{nV}$. Analog fully differential signaling presents many advantages in low-level signal acquisition. First, the signal is protected from the noise coupling paths, which are reference and power supply, and second, by definition, the signal amplitude is double of that in single-ended signals. Differential signals also improve EMI immunity, which directly depends on the return current loop areas in the printed circuit board (PCB). In order to reduce EMI within the analog stage of the PCB, the areas formed by the currents flowing through tracks and the return currents that flow under the tracks have to be minimized. In differential signals, this is achieved easily, as the return current related to the negative terminal has the same magnitude and opposite sign of the one that appears on the positive terminal and, therefore, the net current cancels out. This is only true if the positive and negative terminals have the same length. Therefore, in the PCB design, differential traces have to be parallel. By minimizing the distance between parallel traces, current loop areas are also minimized, resulting in reduced EMI (Figure 4) [5].

High-frequency noise rejection: In seismology, the signal bandwidth is DC to $100 \mathrm{~Hz}$ for the geophone and $20 \mathrm{kHz}$ for the hydrophone. To reject high-frequency noise, low-pass differential RC filters are used before signal amplification. The cutoff frequency is placed at $40 \mathrm{kHz}$, much higher than the signal bandwidth.

PCB design optimizations: The PCB of the ADC module is divided into four layers:

- Top layer: analog stage and low-frequency signals are included on this layer. There are very few high-frequency signals (ADC clock, digital data, etc.) available. They are placed far away from the analog input, thus minimizing the effects of crosstalk and EMI on the data.

- Ground layer: the ground layer is a solid reference without tracks or discontinuities and is the best way to minimize 
return current loop areas [6] when differential signals are used. The analog and digital grounds are common, as

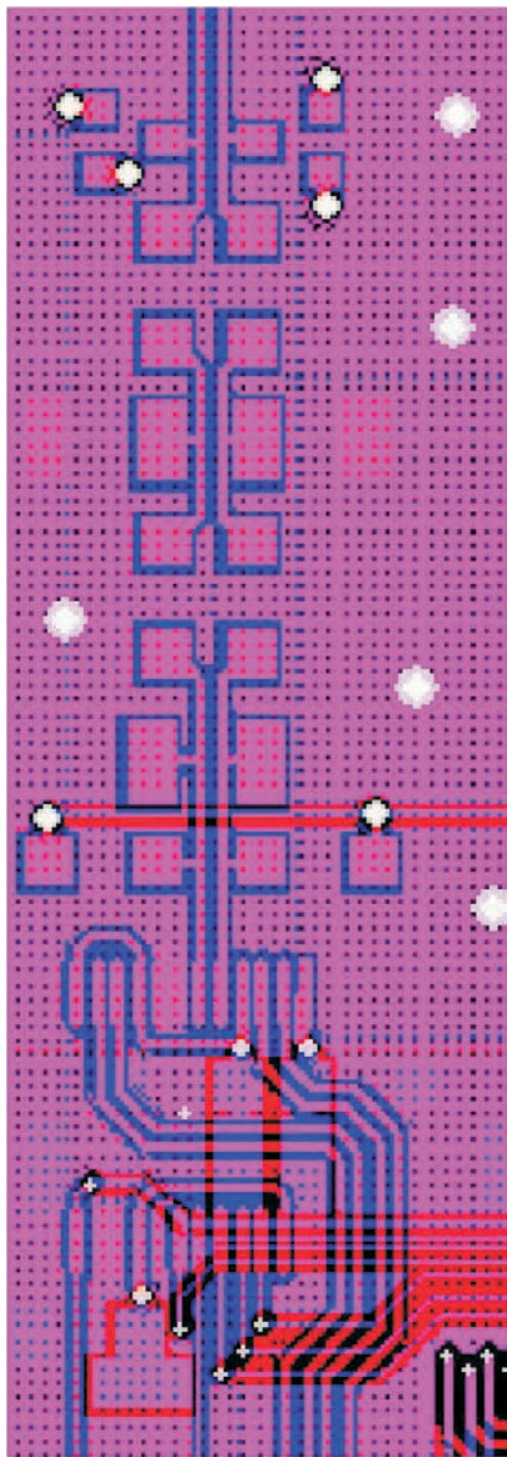

Fig. 4. Differential signal traces of an input channel of the ADC module. the input signal is not referenced to ground.

D Power supply layer: this layer contains power supply tracks as well as a reference plane in order to reduce EMI. The stack-up of the PCB is carried out in such a way that power supply and ground layers face each other, creating a decoupling capacitor that improves noise decoupling to the analog input through the power supply pins.

- Bottom layer: all communication components that use high-frequency signals are placed on this layer, minimizing the EMI and crosstalk onto the analog input.

In the PCB design, all the input analog traces are routed as differential pairs. Therefore, all source and return analog signal currents are constrained to the differential pair route and do not return through the ground plane [5]. Figure 4 shows the analog differential signal traces of an input channel of the PCB.

Each device supply pin includes a $100-\mathrm{nF}$ capacitor for high-frequency noise rejection and a $10-\mu \mathrm{F}$ capacitor for lowfrequency noise rejection. Both capacitors are placed as close as possible to the supply pin, with the lower capacitor placed closer to the pin to reject the noise generated by the trace itself. Furthermore, special care has been taken in choosing the capacitor technology for analog filtering, anti-alias RC network, and the voltage reference of the ADCs, specified by the ADC manufacturer. Figure 5 shows the ADC module implemented.

\section{Acquisition System Tests}

In the last decade, many marine research institutes have designed and implemented acquisition systems for their OBS. However, at present there is a lack of standard procedures for characterization of these systems. Every marine institute provides specifications of their acquisition system, but they do not give any indication of how they have been calculated. To find the performance of the data logger, a series of tests based on IEEE Standard 1057, Standard for Digitizing Waveform Recorders [1], and IEEE Standard 1241, Standard for Terminology and Test Methods for Analog-to-Digital Converters [2], are implemented in LabVIEW [7]. Some of these tests are not direct implementation of the tests included in the standards; however, the parameters are calculated by using the definitions and expressions given in the standards. These tests are the following:

D The discrete Fourier transform (DFT) test,

D The random noise test,

- The crosstalk test, and

D The ADC clock jitter test.

The selected parameters to be calculated take into account the specifications needed to characterize an OBS. In the DFT, crosstalk, and ADC clock jitter tests, a sine wave is applied at the input of the system and a known number of samples are acquired. These samples are used to calculate the desired parameters using the expressions indicated in the standards. In the random noise test, no signal is applied to the input. The signal reconstruction of the acquired data is given by

$$
V_{\text {sample }}=\left(\operatorname{Code}^{*} Q\right)+T[1]
$$

where $Q$ is the code width and $T[1]$ is the first transition level [8] defined by the transfer function of the CS5372 modulator. In the case, Bipolar with true zero

$$
T[1]=-V_{\min }+\frac{Q}{2}
$$

where $V_{\min }$ is the minimum voltage that can be applied to the modulator without an output error [8].

Taking into account that the system under test has a 24-bit resolution and that a noise analysis is necessary, low-noise instruments have to be used to calculate the parameter correctly. In this case, an ultra-low-noise and distortion Stanford Research System (SRS) DS360 function generator is used to generate the

Fig. 5. A/D conversion module 


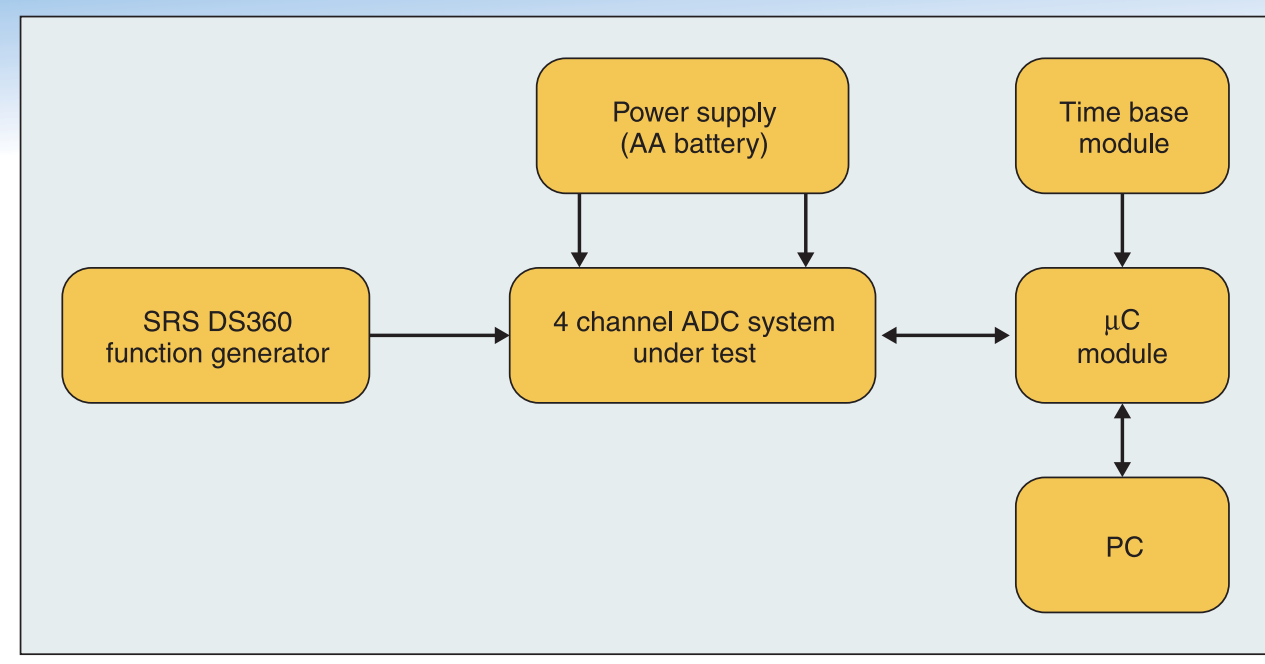

Fig. 6. System setup used in the characterization tests. be acquired. By adjusting the input signal frequency using equation (3), we can almost achieve an integer number of cycles in the record. In practice, due to physical errors such as random noise of the ADC clock, signal generator phase noise and ADC quantization noise, the number of periods present in acquisition will never be an exact integer. The equation that relates the input signal frequen$c y, f$, is

$$
\frac{f}{f_{s}}=\frac{J}{M}
$$

input signals. The DS360 function generator presents a $-110 \mathrm{~dB}$ THD in $5 \mathrm{kHz}$ bandwidth with a minimum $20 \mu \mathrm{V}_{\mathrm{pp}}$ differential amplitude, allowing characterization of the system by using low-input amplitudes, as in real conditions. Another common source of noise coupled to the system is the power supply. Power supplies are often not filtered and are not suitable for low-noise systems. Therefore, we have used batteries to supply the acquisition system, which is the best way to minimize the noise coupling from the source to the system. Figure 6 shows a block diagram of the system set up in the lab in order to carry out the characterization tests.

As a result of impurities in the input signal from the signal generator, there is a leakage of energy from the fundamental frequency to the nearby frequencies, causing spectral leakage in the DFT calculation. Another cause of spectral leakage is discontinuous acquisition of the input signal. To minimize the effect of spectral leakage, an exact integer number of periods of the input signal has to

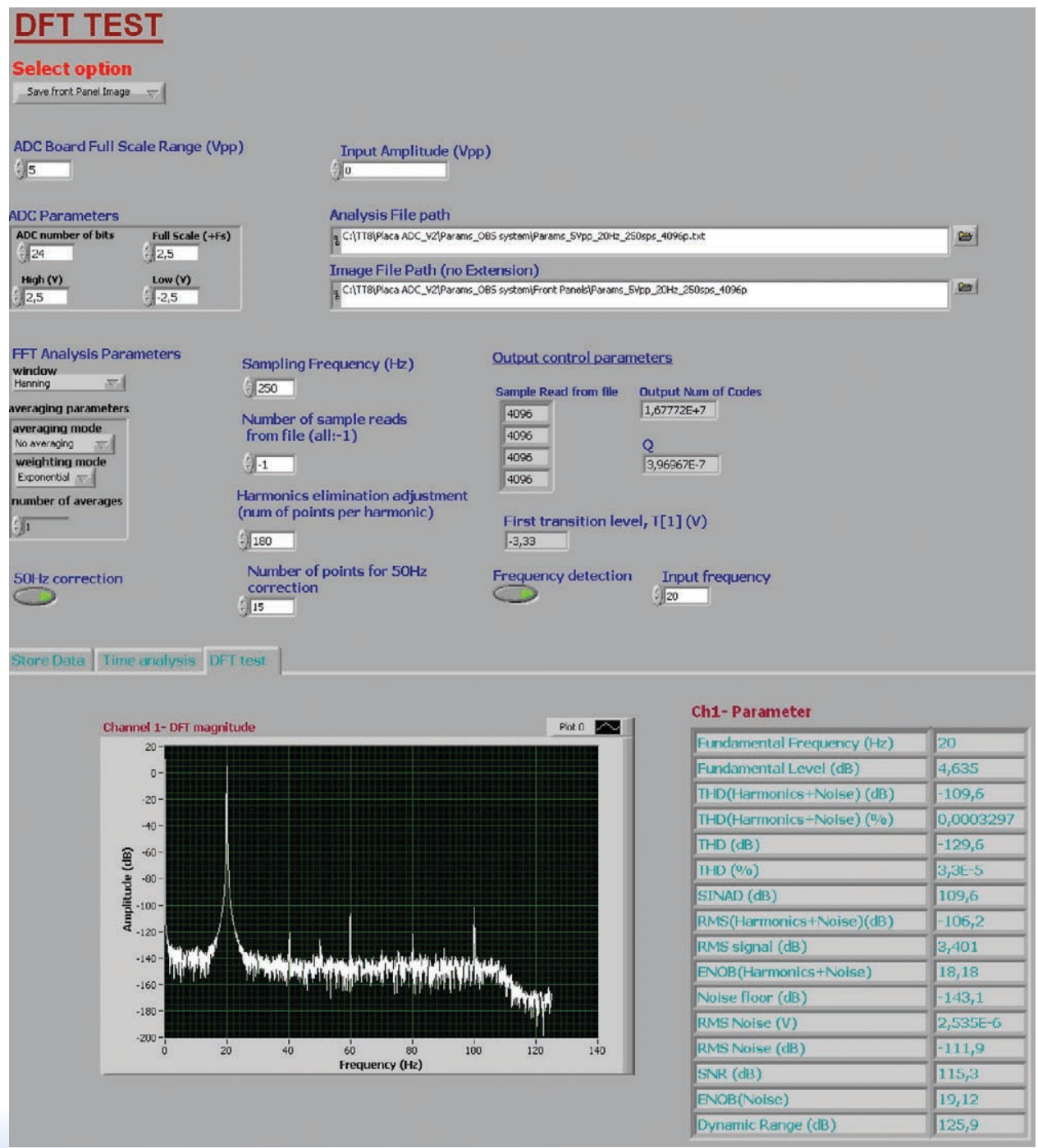

Fig. 7. Front panel of the discrete Fourier transform (DFT) test. 


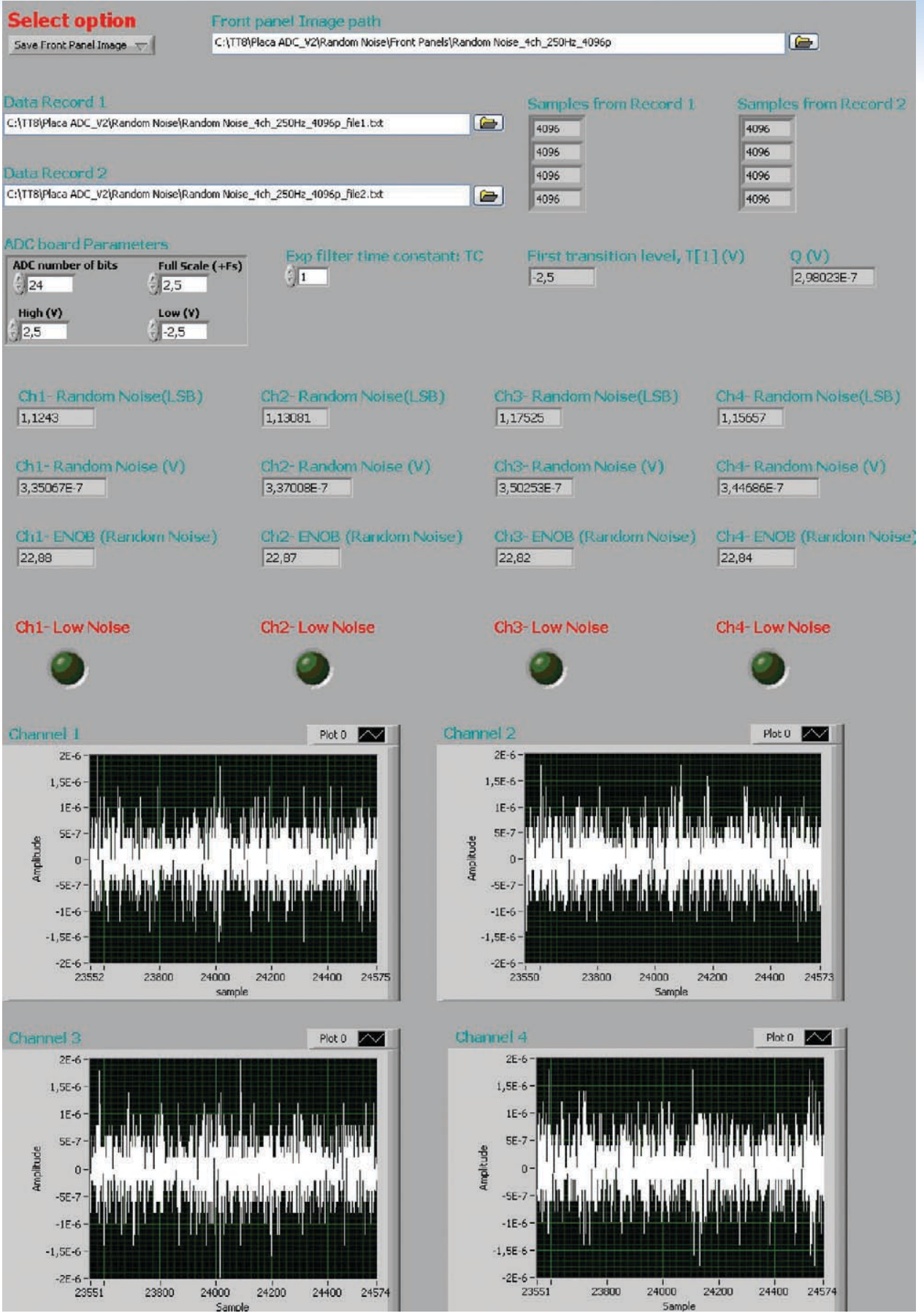

Fig. 8. Front panel of the random noise test when all channels are active.

where $f_{s}$ is the sampling frequency, $M$ is the total samples acquired, and $J$ is an integer prime number to $M$. Moreover, a digital window can be applied to the acquired data. In this case we have used a Hann (Hanning) window, taking into account the type of signal generated in real conditions by the compressedair gun. The test procedure for each test is described below.

\section{The Test Procedures}

DFT test [1], [2]: A sine wave signal with a known amplitude and frequency is applied at the input of the system, and 4,096 samples/channel, with a sampling frequency of 250 samples/s, are acquired and transferred to a PC. A program in LabVIEW that runs in the PC is in charge of data storage in a file. The DFT Analysis option of the characterization software reads the data samples stored previously and represents the DFT of every channel on a separate graph. Noise and distortion parameters are calculated from the DFT components, and results for every channel are given in separate tables. The Save Front Panel Image option allows the user to store the calculated parameters in a file. Figure 7 shows the front panel of the DFT test. The results correspond to one of the channels.

To minimize the effects of spectral leakage and line interference $(50 \mathrm{~Hz})$ coupled to the system through the signal generator on the results, the software includes algorithms to correct these errors before the parameter calculation.

Random noise test [1], [2]:

To calculate the random noise generated by the input amplifier stage of the ADC module, a random noise test is implemented. Figure 8 shows the front panel of the random noise test. All input channels are grounded. Two separate data files of 4,096 samples / channel at 250 samples/s are acquired and stored in a PC. The Random Noise Analysis option of the characterization software reads the data files previously stored, removes the ADC fixed-pattern errors that occur in the same location of successive records by subtracting the data samples, and calculates the noise variance given by:

$$
\sigma^{2}=\frac{1}{2 M} \sum_{n=1}^{M}\left(y_{a n}-y_{b n}\right)^{2}
$$

where $\sigma^{2}$ is the noise variance, $y_{a n}$ and $y_{b n}$ are data samples in the data files, and $\mathrm{M}$ is the record length for every channel. The Save Front Panel Image option allows for storing the final results. 
Crosstalk test [1]: Often calculation of channel crosstalk is a good evaluation of the PCB design of an ADC module. See [2] for terminology, definitions, and meaning of the parameter. Electromagnetic interference (EMI) coupled to the analog input as well as poor distribution of ground and power supply planes have a direct effect on the crosstalk value. In this case, a full-scale sine wave $\left(5 \mathrm{~V}_{\mathrm{pp}}\right)$ is applied to the channel under test while all the other channels are grounded. Four thousand ninety-six samples/channel are acquired and stored in a data file. The Crosstalk Analysis option of the characterization software reads the data file stored previously, and the crosstalk level of the channel under test is given by the ratio between the rms of a spurious signal level coupled and the rms input signal amplitude. In this test, the spurious signal is the signal coupled from the channel under test to all other channels at the input signal frequency.

$$
\text { Crosstalk }(d B)=20 \log \left[\frac{R M S(\text { spurious })}{R M S(\text { signal })}\right]
$$

ADC clock jitter test [1]: The method used to find the ADC clock jitter is based on the calculation of rms noise resulting from clock random errors. Two different input signal frequencies are used to calculate ADC clock jitter: a sine wave of $5 \mathrm{~Hz}$ $\left(f_{1}\right)$ and another one of $90 \mathrm{~Hz}\left(f_{2}\right)$. The input signal amplitude of $2.25 \mathrm{~V}(A)$ remains constant. The rms noise values of $\sigma_{1}$ and $\sigma_{2}$ at both frequencies are calculated using Equation 6:

$$
\sigma=\sqrt{\frac{1}{M} \sum_{n=1}^{M}\left(y_{n}-y_{n}^{\prime}\right)^{2}},
$$

where $y_{n}$ represents the collected samples, $\mathrm{y}_{\mathrm{n}}^{\prime}$ represents the sine-fitted samples of the data, and $M$ is the record length $(M=1,024)$. The upper bound for clock jitter is given by

$$
\sigma_{t}=\frac{\sqrt{\sigma_{2}^{2}-\sigma_{1}^{2}}}{\sqrt{2} \pi f_{2} A}
$$

There are two methods given in [1] for the ADC clock jitter estimation. We have used the above method because error sources such as ADC quantization noise and additive noise do not affect the result given in the ADC clock jitter estimation. The subtraction in Equation 7 eliminates their effect, providing a better estimation of the jitter [9].

\section{Results}

The implemented tests described in the previous section are used to find the specifications of the acquisition system before carrying out an active seismic experiment. Noise parameters are calculated by using a $20-\mu \mathrm{V}_{\mathrm{pp}}$ differential input sine wave minimizing the noise coupled from the signal generator to the system. This constitutes a realistic case, as in active seismology, where the signal level detected by the instrument is very low.
The specifications of the data logger show that the dynamic range of all the channels is about $129 \mathrm{~dB}$, slightly below the ADC dynamic range at the same sampling rate $(130 \mathrm{~dB}$ at 103 $\mathrm{Hz}$ ), while the resolution of the system is about 21.4 bits as a result of noise. In the test implemented, the dynamic range is given by equation (8):

$$
D R(d B)=20 \log \left[\frac{R M S\left(A_{\text {fullsale }}\right)}{R M S(\text { noise })}\right]
$$

where $A_{\text {fullscale }}$ is the amplitude of the full scale sine wave applied at the input. The random noise is about 1.1 least significant bits (LSBs), an rms value, which corresponds to about 320 $\mathrm{nV}$. A channel crosstalk of $-147 \mathrm{~dB}$ shows a good PCB design and EMI immunity. We also note that channel 1 presents slightly lower performance because of its higher noise level. This channel is dedicated to the hydrophone and has a different analog filter than the rest of channels. The performance of the input analog filter of this channel has to be improved in order to minimize the noise level. The ADC clock jitter is rated at $76.5 \pm 3.3 \mathrm{~ns}$ with a $95 \%$ confidence level. Complete results of the characterization tests for all of the channels can be found in [4].

The OBS prototype data has been compared with OBSs from the IFM-Geomar Institute, Germany, and from Scripps Oceanographic Institute in real environmental conditions with satisfactory results in 2006 [4] and 2008. OBSs were placed in the same location, an air gun was used as the acoustic source, and the acquired data from different OBSs were compared after signal processing in the lab [4].

\section{Conclusions}

We explained the design and test of an acquisition system built for marine seismology. A series of optimizations are carried out in the design of the data logger as well as in its PCB implementation to maximize the $S / N$ ratio. The characterization tests implemented help to carry out optimizations of the system before being used in real environmental conditions and define the specifications of the final instrument. The SRS DS360 function generator has shown appropriate features for the characterization of high-resolution waveform recorders. Low-amplitude signals have to be used to minimize the noise coupled from the generator to the system. Comparing the specifications given in the previous section with similar systems built for OBSs, we can conclude that the noise level is low enough, and with 2 months of autonomy, this data logger can be used for mid-term marine seismology.

\section{References}

[1] IEEE Std, 1057, Apr. 2008.

[2] IEEE Std, 1241, Dec. 2000.

[3] J. Bialas and E.R. Flueh, "Ocean bottom seismometers," Sea Technology Magazine, vol. 40, (no. 4), pp. 41-46, Apr. 1999.

[4] S. Shariat-Panahi, A. Mànuel, F. Alegria, X. Roset, A. Bermúdez, and V. Sallares, "Design, characterization and calibration of a short 
period ocean bottom seismometer (OBS)," in Proc. $I^{2}$ MTC 2008, May 2008, pp. 495-500.

[5] D. Brooks, Signal Integrity Issues and Printed Circuit Board Design Upper Saddle River, NJ: Prentice Hall, 2003.

[6] H. Johnson and M. Graham, High Speed Digital Design, Saddle River, NJ: Prentice Hall, 1993.

[7] S. Shariat-Panahi, F. Alegria, and A. Mànuel, "Characterization of a high resolution acquisition system for marine geophysical applications," in Proc. IMTC 2006, 2006, pp. 1317-1320.

[8] F.C. Alegria and A.C. Serra, "ADC transfer function types-A review," Journal of Computer Standards \& Interfaces, vol. 28, (no. 5), pp. 553-559, Jun 2006.

[9] S. Shariat Panahi, F. Corrêa Alegria, A. Manuel, and A. Cruz Serra, "Time base error characterization of a data recorder aimed for marine seismology," in Proc. IMTC 2007, 2007, p. 18.

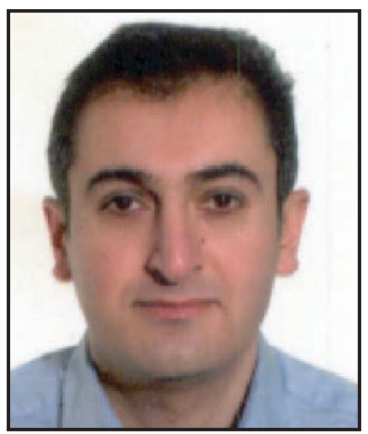

Shahram Shariat-Panahi (shahram.shariat@upc.edu) received his Ph.D. in electronic engineering in 2007 from the Technical University of Catalonia (UPC) in Barcelona, Spain. At present he is an associate professor in the Electronic Engineering department at UPC. His research areas include design and implementation of high-resolution data acquisition systems, characterization of ADCs and waveform record- ers, design and implementation of smart sensors for underwater observatories, and underwater acoustics.

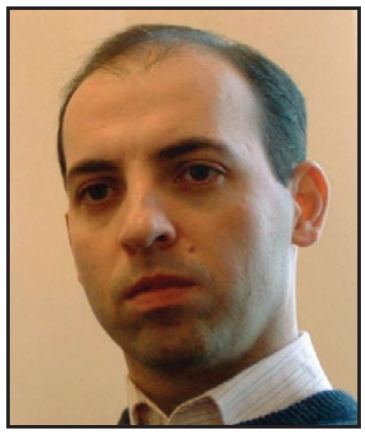

Francisco Corrêa Alegria (falegria@lx.it.pt) received his Ph.D. in electrical engineering and computers in 2002 from the Instituto Superior Tecnico (IST), Technical University of Lisbon. Since 1997, he has been a member of the teaching and research staff at IST. His current research interests include analog-to-digital converter characterization techniques, automatic measurement systems, and computer vision.

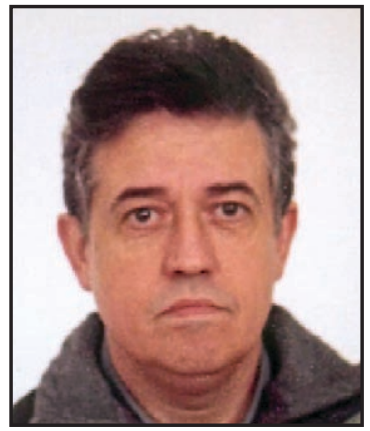

Antoni Mànuel Làzaro (antoni. manuel@upc.edu) received his Ph.D. in telecommunications engineering from the UPC in 1996. Since 1988, he has been an associate professor in the Department of Electronic Engineering at UPC. At present he is the director of the SARTI (Remote acquisition systems and data processing) research group. 